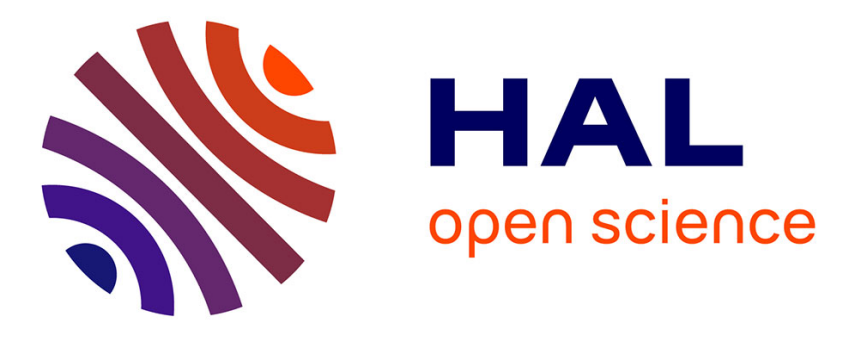

\title{
Unified Model for Assessing Checkpointing Protocols at Extreme-Scale
}

George Bosilca, Aurélien Bouteiller, Elisabeth Brunet, Franck Cappello, Jack

Dongarra, Amina Guermouche, Thomas Herault, Yves Robert, Frédéric

Vivien, Dounia Zaidouni

\section{To cite this version:}

George Bosilca, Aurélien Bouteiller, Elisabeth Brunet, Franck Cappello, Jack Dongarra, et al.. Unified Model for Assessing Checkpointing Protocols at Extreme-Scale. Concurrency and Computation: Practice and Experience, 2013, 26 (17), pp.2727-2810. 10.1002/cpe.3173 . hal-00908447

\section{HAL Id: hal-00908447 https://hal.inria.fr/hal-00908447}

Submitted on 23 Nov 2013

HAL is a multi-disciplinary open access archive for the deposit and dissemination of scientific research documents, whether they are published or not. The documents may come from teaching and research institutions in France or abroad, or from public or private research centers.
L'archive ouverte pluridisciplinaire HAL, est destinée au dépôt et à la diffusion de documents scientifiques de niveau recherche, publiés ou non, émanant des établissements d'enseignement et de recherche français ou étrangers, des laboratoires publics ou privés. 


\title{
Unified Model for Assessing Checkpointing Protocols at Extreme-Scale
}

\author{
George Bosilca ${ }^{1}$, Aurélien Bouteiller ${ }^{1}$, Elisabeth Brunet ${ }^{2}$, Franck Cappello ${ }^{3}$, Jack \\ Dongarra $^{1}$, Amina Guermouche ${ }^{4 *}$, Thomas Herault ${ }^{1}$, Yves Robert $^{1,4}$, Frédéric Vivien ${ }^{4}$, \\ and Dounia Zaidouni ${ }^{4}$ \\ ${ }^{1}$ University of Tennessee Knoxville, USA \\ ${ }^{2}$ Telecom SudParis \\ ${ }^{3}$ INRIA \& University of Illinois at Urbana Champaign, USA \\ ${ }^{4}$ Ecole Normale Supérieure de Lyon \& INRIA, France
}

\begin{abstract}
SUMMARY
In this paper, we present a unified model for several well-known checkpoint/restart protocols. The proposed model is generic enough to encompass both extremes of the checkpoint/restart space, from coordinated approaches to a variety of uncoordinated checkpoint strategies (with message logging). We identify a set of crucial parameters, instantiate them and compare the expected efficiency of the fault tolerant protocols, for a given application/platform pair. We then propose a detailed analysis of several scenarios, including some of the most powerful currently available HPC platforms, as well as anticipated Exascale designs. The results of this analytical comparison are corroborated by a comprehensive set of simulations. Altogether, they outline comparative behaviors of checkpoint strategies at very large scale, thereby providing insight that is hardly accessible to direct experimentation. Copyright (c) 2010 John Wiley \& Sons, Ltd.
\end{abstract}

Received ..

KEY WORDS: Checkpoint/restart, coordinated checkpoint, hierarchical checkpoint with message logging, checkpointing waste optimization problem

\section{INTRODUCTION}

A significant research effort is focusing on the characteristics, features, and challenges of High Performance Computing (HPC) systems capable of reaching the Exaflop performance mark [1,2]. The portrayed Exascale systems will necessitate billion way parallelism, resulting not only in a massive increase in the number of processing units (cores), but also in terms of computing nodes.

Considering the relative slopes describing the evolution of the reliability of individual components on one side, and the evolution of the number of components on the other side, the reliability of the entire platform is expected to decrease, due to probabilistic amplification. Executions of large parallel applications on these systems will have to tolerate a higher degree of errors and failures than in current systems. Preparation studies forecast that standard fault tolerance approaches (e.g., coordinated checkpointing on parallel file system) will lead to unacceptable overheads at Exascale. Thus, it is not surprising that improving fault tolerance techniques is one of the main recommendations isolated by these studies $[1,2]$.

\footnotetext{
${ }^{*}$ Correspondence to: Journals Production Department, John Wiley \& Sons, Ltd, The Atrium, Southern Gate, Chichester, West Sussex, PO19 8SQ, UK. 
In this paper we focus on techniques tolerating the effect of detected errors that prevent successful completion of the application execution. Undetected errors, also known as silent errors, are out-ofscope of this analysis. There are two main ways of tolerating process crashes, without undergoing significant application code refactoring: replication and rollback recovery. An analysis of replication feasibility for Exascale systems was presented in [3]. In this paper we focus on rollback recovery, and more precisely on the comparison of checkpointing protocols.

There are three main families of checkpointing protocols: (i) coordinated checkpointing; (ii) uncoordinated checkpointing with message logging; and (iii) hierarchical protocols mixing coordinated checkpointing and message logging. The key principle in all these checkpointing protocols is that all data and states necessary to restart the execution are regularly saved in process checkpoints. Depending on the protocol, these checkpoints are or are not guaranteed to form consistent recovery lines. When a failure occurs, appropriate processes rollback to their last checkpoints and resume execution.

Each protocol family has serious drawbacks. Coordinated checkpointing and hierarchical protocols suffer a waste in terms of computing resources, whenever living processes have to rollback and recover from a checkpoint in order to tolerate failures. These protocols may also lead to I/O congestion when too many processes are checkpointing at the same time. Message logging increases memory consumption, checkpointing time, and slows down failure-free execution when messages are logged. Our objective is to identify which protocol delivers the best performance for a given application on a given platform. While several criteria could be considered to make such a selection, we focus on the most widely used metric, namely, the expectation of the total parallel execution time.

Fault-tolerant protocols have different overheads in fault-free and recovery situations. These overheads depend on many factors (type of protocols, application characteristics, system features, etc.) that introduce complexity and limit the scope of experimental comparisons conducted in the past $[4,5]$. In this paper, we approach the fault tolerant protocol comparison from an analytical perspective. Our objective is to provide an accurate performance model covering the most suitable rollback recovery protocols for HPC. This model captures many optimizations proposed in the literature, but can also be used to explore the effects of novel optimizations, and to highlight the most critical parameters to be considered when evaluating a protocol.

The main contributions of this paper are: (1) a comprehensive model that captures many rollback recovery protocols, including coordinated checkpoint, uncoordinated checkpoint, and the composite hierarchical hybrids; (2) a closed-form formula for the waste of computing resources incurred by each protocol. This formula is the key to assessing existing and new protocols, and constitutes the first tool that can help the community to compare protocols at very large scale, and to guide design decisions for given application/platform pairs; and (3) an instantiation of the model on several realistic scenarios involving state-of-the-art and future Exascale platforms, thereby providing practical insight and guidance.

This paper is organized as follows. Section 2 details the characteristics of available rollback recovery approaches, and the tradeoff they impose on failure-free execution and recovery. We also discuss related work in this section. In Section 3, we describe our model that unifies coordinated rollback recovery approaches, and effectively captures coordinated, partially and totally uncoordinated approaches as well as many of their optimizations. We then use the model to analytically assess the performance of rollback recovery protocols. We instantiate the model with realistic scenarios in Section 4, and we present corresponding results in Section 5. These results are corroborated by a set of simulations (Section 6), demonstrating the accuracy of the proposed unified analytical model. Finally, we conclude and present perspectives in Section 7.

\section{BACKGROUND}

\subsection{Rollback Recovery Strategies}

Rollback recovery addresses permanent (fail-stop) process failures, in the sense that a process reached a state where either it cannot continue for physical reasons or it detected that the current 
state has been corrupted and further continuation of the current computation is worthless. In order to mitigate the cost of such failures, processes periodically save their state on persistent memory (remote node, disk, ...) by taking checkpoints. In this paper, we consider only the case of fault tolerant protocols that provide a consistent recovery, immune to the domino effect [6]. This can be achieved by two approaches; On one extreme, coordinating checkpoints, where after a failure, the entire application rolls back to a known consistent global state; On the opposite extreme, message logging, which allows for independent restart of failed processes but logs supplementary state elements during the execution to drive a directed replay of the recovering processes. The interested reader can refer to [6] for a comprehensive survey of message logging approaches, and to [7] for a description of the most common algorithm for checkpoint coordination. Although the uncoordinated nature of the restart in message logging improves recovery speed compared to the coordinated approach (during the replay, all incoming messages are available without jitter, most emissions are discarded and other processes can continue their progress until they need to synchronize with replaying processes) [4], the logging of message payload incurs a communication overhead and an increases in the size of checkpoints directly influenced by the communication intensity of the application [8]. Recent advances in message logging [9, 10, 11] have led to composite algorithms, called hierarchical checkpointing, capable of partial coordination of checkpoints to decrease the cost of logging, while retaining message logging capabilities to remove the need for a global restart. These hierarchical schemes partition the application processes in groups. Each group checkpoints independently, but processes belonging to the same group coordinate their checkpoints and recovery. Communications between groups continue to incur payload logging. However, because processes belonging to a same group follow a coordinated checkpointing protocol, the payload of messages exchanged between processes within the same group is not required to be logged.

The optimizations driving the choice of the size and shape of groups are varied. A simple heuristic is to checkpoint as many processes as possible, simultaneously, without exceeding the capacity of the I/O system. In this case, groups do not checkpoint in parallel. Groups can also be formed according to hardware proximity or communication patterns. In such approaches, there may be opportunity for several groups to checkpoint concurrently. The model we propose captures the intricacies of all such strategies, thereby also representing both extremes, coordinated and uncoordinated checkpointing. In Section 4, we describe the meaningful parameters to instantiate these various protocols for a variety of platforms and applications, taking into account the overhead of message logging, and the impact of grouping strategies.

\subsection{Related work}

The question of optimal period of checkpoint for sequential jobs (or parallel jobs undergoing coordinated checkpointing) has seen many studies presenting different order of estimates: see [12, 13], and [14, 15] that consider Weibull distributions, or [16] that considers parallel jobs. This is critical to extract the best performance of any rollback-recovery protocol. However, although we use the same approach to find the optimal checkpoint interval, we focus our study on the comparison of different protocols that were not captured by previous models.

The literature proposes different works $[17,18,19,20,21]$ on the modeling of coordinated checkpointing protocols. [22] focuses on refining failure prediction; [18] and [17] focus on the usage of available resources: some may be kept as backup in order to replace the down ones, and others may be even shutdown in order to decrease the failure risk or to prevent storage consumption by saving fewer checkpoint snapshots. [21] proposes a scalability model where they evaluate the impact of failures on application performance. A significant difference with these works lies in the inclusion of several new parameters to refine the model.

The uncoordinated and hierarchical checkpointing have been less frequently modeled. [23] models periodic checkpointing on fault-aware parallel tasks that do not communicate. From our point of view, this specificity does not match the uncoordinated checkpointing with message logging we consider. In this paper, we consistently address all the three families of checkpointing protocols: coordinated, uncoordinated, and hierarchical ones. To the best of our knowledge, it is the first attempt at providing a unified model for this large spectrum of protocols. 


\section{MODEL AND ANALYTICAL ASSESSMENT}

In this section, we discuss the unified model, together with the closed-form formulas for the waste optimization problem. We start with the description of the abstract model (Section 3.1). Processors are partitioned into $G$ groups, where each group checkpoints independently and periodically. To help follow the technical derivation of the waste, we start with one group (Section 3.2) before tackling the general problem with $G \geq 1$ groups (Section 3.3), first under simplified assumptions, before tackling last the fully general model, which requires three additional parameters (payload overhead, faster execution replay after a failure, and increase in checkpoint size due to logging). We end up with a complicated formula that characterizes the waste of resources due to checkpointing. This formula can be instantiated to account for checkpointing protocols, see Section 4 for examples. Note that in all scenarios, we model the behavior of tightly coupled applications, meaning that no computation can progress on the entire platform as long as the recovery phase of a group with a failing processor is not completed.

\subsection{Abstract model}

In this section, we detail the main parameters of the model. We consider an application that executes on $p_{\text {total }}$ processors.

Units- To avoid introducing several conversion parameters, we represent all the parameters of the model in seconds. The failure inter-arrival times, the duration of a downtime, checkpoint, or recovery are all expressed in seconds. Furthermore, we assume (without loss of generality) that one work unit is executed in one second, when all processors are computing at full rate. One work-unit may correspond to any relevant application-specific quantity. When a processor is slowed-down by another activity related to fault-tolerance (writing checkpoints to stable storage, logging messages, etc.), one work-unit takes longer than a second to complete.

Failures and $M T B F$ - The platform consists of $p_{\text {total }}$ identical processors. We use the term "processor" to indicate any individually scheduled compute resource (a core, a socket, a cluster node, etc) so that our work is agnostic to the granularity of the platform. These processors are subject to failures. Exponential failures are widely used for theoretical studies, while Weibull or log-normal failures are representative of the behavior of real-world platforms [24, 25, 26, 27]. The mean time between failures of a given processor is a random variable with mean $(M T B F) \mu$ (expressed in seconds). Given the failure distribution of one processor, it is difficult to compute, or even approximate, the failure distribution of a platform with $p_{t o t a l}$ processors, because it is the superposition of $p_{\text {total }}$ independent and identically distributed distributions (with a single processor). However, there is an easy formula for the MTBF of that distribution, namely $\mu_{p}=\frac{\mu}{p_{\text {total }}}$.

In our theoretical analysis, we do not assume to know the failure distribution of the platform, except for its mean value (the MTBF). Nevertheless, consider any time-interval $\mathcal{I}=[t, t+T]$ of length $T$ and assume that a failure strikes during this interval. We can safely state that the probability for the failure to strike during any sub-interval $\left[t^{\prime}, t^{\prime}+X\right] \subset \mathcal{I}$ of length $X$ is $\frac{X}{T}$. Similarly, we state that the expectation of the time $m$ at which the failure strikes is $m=t+\frac{T}{2}$. Neither of these statements rely on some specific property of the failure distribution, but instead are a direct consequence of averaging over all possible interval starting points, that will correspond to the beginning of checkpointing periods, and that are independent of failure dates.

Tightly-coupled application- We consider a tightly-coupled application executing on the $p_{\text {total }}$ processors. Inter-processor messages are exchanged throughout the computation, which can only progress if all processors are available. When a failure strikes a processor, the application is missing one resource for a certain period of time of length $D$, the downtime. Then, the application recovers from the last checkpoint (recovery time of length $R$ ) before it re-executes the work done since that checkpoint and up to the failure. Under a hierarchical scenario, the useful work resumes only when the faulty group catches up with the overall state of the application at failure time. Many scientific applications are tightly-coupled and obey such a recovery scheme. Typically, the tightly-coupled application will be an iterative application with a global synchronization point at the end of each iteration. However, the fact that inter-processor information is exchanged continuously or at given 
synchronization steps (as in BSP-like models) is irrelevant: in steady-state mode, all processors must be available concurrently for the execution to actually progress. While the tightly-coupled assumption may seem very constraining, it captures the fact that processes in the application depend on each other and exchange messages at a rate exceeding the periodicity of checkpoints, preventing independent progress.

Blocking or non-blocking checkpoint- There are various scenarios to model the cost of checkpointing, so we use a flexible model, with several parameters to specify. The first question is whether checkpoints are blocking or not. On some architectures, we may have to stop executing the application before writing to the stable storage where the checkpoint data is saved; in that case checkpoint is fully blocking. On other architectures, checkpoint data can be saved on the fly into a local memory before the checkpoint is sent to the stable storage, while computation can resume progress; in that case, checkpoints can be fully overlapped with computations. To deal with all situations, we introduce a slow-down factor $\alpha$ : during a checkpoint of duration $C$, the work that is performed is $\alpha C$ work units, instead of $C$ work-units if only computation takes place. In other words, $(1-\alpha) C$ work-units are wasted due to checkpoint jitter perturbing the progress of computation. Here, $0 \leq \alpha \leq 1$ is an arbitrary parameter. The case $\alpha=0$ corresponds to a fully blocking checkpoint, while $\alpha=1$ corresponds to a fully overlapped checkpoint, and all intermediate situations can be represented.

Periodic checkpointing strategies- For the sake of clarity and tractability, we focus on periodic scheduling strategies where checkpoints are taken at regular intervals, after some fixed amount of work-units have been performed. This corresponds to an infinite-length execution partitioned into periods of duration $T$. Without loss of generality, we partition $T$ into $T=W+C$, where $W$ is the amount of time where only computations take place, while $C$ corresponds to the amount of time where checkpoints are taken.

Let TIME $E_{\text {base }}$ be the application execution time without any fault tolerance mechanism and without failures. If we assume that TIME $\mathrm{FF}_{\mathrm{F}}$ is the execution time when checkpoints are introduced and WASTE $[F F]$ is the waste due to checkpoints, TIME ${ }_{\text {base }}$ would be equal to TIMEFF $_{\mathrm{FF}}$ minus the waste due to checkpoints, thus:

$$
\left.\left(1-\text { WASTE}_{F F} F\right]\right) \operatorname{TiME}_{\mathrm{FF}}=\text { TIME }_{\text {base }}
$$

With the same idea, if we assume that TIME $\mathrm{final}_{\text {in }}$ is the time needed to complete the execution with failures and fault tolerance techniques:

$$
\left(1-\text { WASTE}_{\text {fail }]}\right) \text { TIME }_{\text {final }}=\text { TIMEFF }_{\text {F }}
$$

By replacing the equation 2 in the equation 1 and if we assume that WASTE is the total waste:

$$
\left.(1-\text { WASTE }[F F])\left(1-\text { WASTE}_{\text {fail }}\right]\right) \operatorname{TIME}_{\text {final }}=\text { TIME }_{\text {base }}
$$

We define WASTE as being the amount of time not performing useful computations,

$$
\text { WASTE }=\left(\text { TIME }_{\text {final }}-\text { TIME }_{\text {base }}\right) / \text { TIME }_{\text {final }}
$$

Finally, we deduce the following formula for the global waste:

$$
\text { WASTE }=1-(1-\text { WASTE }[F F])(1-\text { WASTE }[\text { fail }])
$$

If not slowed down for other reasons by the fault tolerant protocol (Section 3.3), the total amount of work units that are executed during a period of length $T$ is thus WORK $=W+\alpha C$ (recall that there is a slow-down due to the overlap). In a failure-free environment, the waste of computing resources due to checkpointing is

$$
\text { WASTE }[F F]=\frac{T-\text { WORK }}{T}=\frac{(1-\alpha) C}{T}
$$

As expected, if $\alpha=1$ there is no overhead, but if $\alpha<1$ (actual slowdown, or even blocking if $\alpha=0$ ), checkpointing comes with a price in terms of performance degradation. 
For the time being, we do not further quantify the length of a checkpoint, which is a function of several parameters. Instead, we proceed with the abstract model. We envision several scenarios in Section 4, only after setting up the formula for the waste in a general context.

Processor groups- As described above, we assume that the platform is partitioned into $G$ groups of the same size. Each group contains $q$ processors, hence $p_{\text {total }}=G q$. When $G=1$, we speak of a coordinated scenario, and we simply write $C, D$ and $R$ for the duration of a checkpoint, downtime and recovery. When $G \geq 1$, we speak of a hierarchical scenario. Each group of $q$ processors checkpoints independently and sequentially in time $C(q)$. Similarly, we use $D(q)$ and $R(q)$ for the durations of the downtime and recovery. Of course, if we set $G=1$ in the (more general) hierarchical scenario, we retrieve the value of the waste for the coordinated scenario. As already mentioned, we derive a general expression for the waste for both scenarios, before further specifying the values of $C(q), D(q)$, and $R(q)$ as a function of $q$ and the various architectural parameters under study.

\subsection{Waste for the coordinated scenario $(G=1)$}

The goal of this section is to quantify the expected waste in the coordinated scenario where $G=1$. The waste is the fraction of time that the processors do not compute at full rate, either because they are checkpointing, or because they are recovering from a failure. Recall that we write $C, D$, and $R$ for the checkpoint, downtime, and recovery using a single group of $p_{\text {total }}$ processors. We obtain the following equation for the waste, which we explain briefly below explanation is available to the reader and illustrate with Figure 1:

(a)
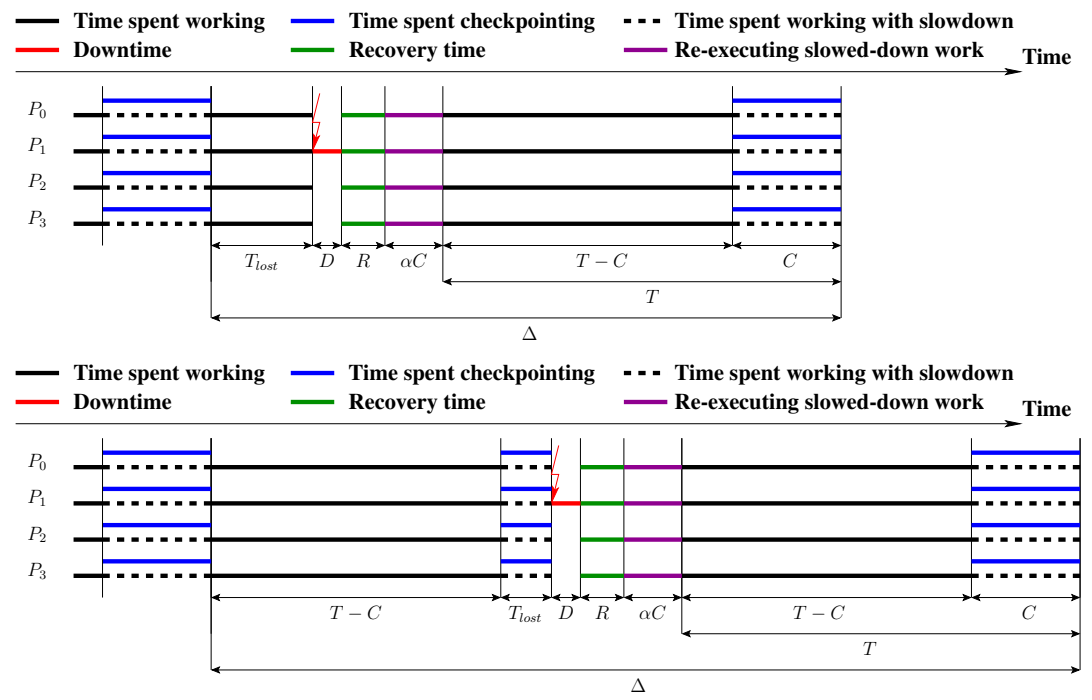

Figure 1. Coordinated checkpoint: illustrating the waste when a failure occurs (a) during the work phase; and (b) during the checkpoint phase.

$$
\begin{aligned}
\text { WAste }[F F]= & \frac{(1-\alpha) C}{T} \\
\text { WAste }[\text { fail }]= & \frac{1}{\mu_{p}}(R+D+ \\
& \frac{T-C}{T}\left[\alpha C+\frac{T-C}{2}\right] \\
+ & \left.\frac{C}{T}\left[\alpha C+T-C+\frac{C}{2}\right]\right)
\end{aligned}
$$


- (7) is the portion of the execution lost in checkpointing, even during a fault-free execution, see Equation (6).

- (9) is the overhead of the execution time due to a failure during work interval $(T-C)$ (see Figure 1(a)).

- (10) is the overhead due to a failure during a checkpoint (see Figure 1(b)).

After simplification of Equations (7) to (10), we get:

$$
\mathrm{WASTE}[\text { fail }]=\frac{1}{\mu_{p}}\left(D+R+\frac{T}{2}+\alpha C\right)
$$

Plugging this value back into Equation (5) leads to:

$$
\text { WASTE }_{\text {coord }}=1-\left(1-\frac{(1-\alpha) C}{T}\right)\left(1-\frac{1}{\mu_{p}}\left(D+R+\frac{T}{2}+\alpha C\right)\right)
$$

We point out that Equation (12) is valid only when $T \ll \mu_{p}$ : indeed, we made a first-order approximation when implicitly assuming that we do not have more than one failure during the same period. This hypothesis is required to allow the expression of the model in a closed form. In fact, the number of failures during a period of length $T$ can be modeled as a Poisson process of parameter $\frac{T}{\mu_{p}}$; the probability of having $k \geq 0$ failures is $\frac{1}{k !}\left(\frac{T}{\mu_{p}}\right)^{k} e^{-\frac{T}{\mu_{p}}}$. Hence the probability of having two or more failures is $\pi=1-\left(1+\frac{T}{\mu_{p}}\right) e^{-\frac{T}{\mu_{p}}}$. Enforcing the constraint $T \leq 0.1 \mu_{p}$ leads to $\pi \leq 0.005$, hence a valid approximation when capping $T$ to that value. Indeed, we have overlapping faults every 200 periods in average, so that our model is accurate for $99.5 \%$ of the checkpointing segments, hence it is quite reliable.

In addition to the previous constraint, we must enforce the condition $C \leq T$, by construction of the periodic checkpointing policy. Without the constraint $C \leq T \leq 0.1 \mu_{p}$, the optimal checkpointing period $\mathbb{T}^{*}$ that minimizes the expected waste in Equation (12) is $\mathbb{T}^{*}=$ $\sqrt{2(1-\alpha)(\mu-(D+R)) C}$. However, this expression for $\mathbb{T}^{*}$ (which is known as Young's approximation [12] when $\alpha=0$ ) may well be out of the admissible range. Finally, note that the optimal waste may never exceed 1 , since it represents the fraction of time that is "wasted". In this latter case, the application no longer makes progress.

\subsection{Waste for the hierarchical scenario $(G \geq 1)$}

In this section, we compute the expected waste for the hierarchical scenario. We have $G$ groups of $q$ processors, and we let $C(q), D(q)$, and $R(q)$ be the duration of the checkpoint, downtime, and recovery for each group. We assume that the checkpoints of the $G$ groups take place in sequence within a period (see Figure 2(a)). We start by generalizing the formula obtained for the coordinated scenario before introducing several new parameters to the model.

3.3.1. Generalizing previous scenario with $G \geq 1$ We obtain the following intricate formula for the waste, which we illustrate with Figure 2 and the discussion below:

$$
\text { WASTE }_{\text {hier }}=1-\left(1-\frac{T-\text { WORK }}{T}\right)\left(1-\frac{1}{\mu_{p}}(D(q)+R(q)+\text { RE-EXEC })\right)
$$

$$
\text { WORK }=T-(1-\alpha) G C(q)
$$




$$
\begin{aligned}
& \text { RE-EXEC }= \\
& \begin{array}{l}
\frac{T-G C(q)}{T} \frac{1}{G} \sum_{g=1}^{G}\left[(G-g+1) \alpha C(q)+\frac{T-G C(q)}{2}\right] \\
+\frac{G C(q)}{T} \frac{1}{G^{2}} \sum_{g=1}^{G}[ \\
\quad \sum_{s=0}^{g-2}(G-g+s+2) \alpha C(q)+T-G C(q) \\
+G \alpha C(q)+T-G C(q)+\frac{C(q)}{2} \\
\left.+\sum_{s=1}^{G-g}(s+1) \alpha C(q)\right]
\end{array}
\end{aligned}
$$

- The first term in Equation (13) represents the overhead due to checkpointing during a fault-free execution (same reasoning as in Equation (6)), and the second term the overhead incurred in case of failure.

- (14) provides the amount of work units executed within a period of length $T$.

- (15) represents the time needed for re-executing the work when the failure happens in a work-only area, i.e., during the first $T-G C(q)$ seconds of the period (see Figure 2(a)).

- (16) deals with the case where the fault happens during a checkpoint, i.e. during the last $G C(q)$ seconds of the period (hence the first term that represents the probability of this event). We distinguish three cases, depending upon what group was checkpointing at the time of the failure:

- (17) is for the case when the fault happens before the checkpoint of group $g$ (see Figure 2(b)).

- (18) is for the case when the fault happens during the checkpoint of group $g$ (see Figure 2(c)).

- (19) is the case when the fault happens after the checkpoint of group $g$, during the checkpoint of group $g+s$, where $g+1 \leq g+s \leq G$ (See Figure 2(d)).

Of course this expression reduces to Equation (12) when $G=1$. Just as for the coordinated scenario, we enforce the constraint

$$
G C(q) \leq T \leq 0.1 \mu_{p}
$$

The first condition is by construction of the periodic checkpointing policy, and the second is to enforce the validity of the first-order approximation (at most one failure per period).

3.3.2. Refining the model We introduce three new parameters to refine the model when the processors have been partitioned into several groups. These parameters are related to the impact of message logging on execution, re-execution, and checkpoint image size, respectively.

Impact of message logging on execution and re-execution- With several groups, inter-group messages need to be stored in local memory as the execution progresses, and event logs must be stored in reliable storage, so that the recovery of a given group, after a failure, can be done independently of the other groups. This induces an overhead, which we express as a slowdown of the execution rate: instead of executing one work-unit per second, the application executes only $\lambda$ work-units, where $0<\lambda<1$. Typical values for $\lambda$ are said to be $\lambda \approx 0.98$, meaning that the overhead due to payload messages is only a small percentage [10,28].

On the contrary, message logging has a positive effect on re-execution after a failure, because inter-group messages are stored in memory and directly accessible after the recovery. Our model accounts for this by introducing a speed-up factor $\rho$ during the re-execution. Typical values for $\rho$ lie in the interval $[1 ; 2]$, meaning that re-execution time can be reduced by up to half for some applications [4].

Fortunately, the introduction of $\lambda$ and $\rho$ is not difficult to account for in the expression of the expected waste: in Equation (13), we replace WORK by $\lambda$ WORK and RE-EXEC by $\frac{\text { RE-EXEC }}{\rho}$ and 
(a)

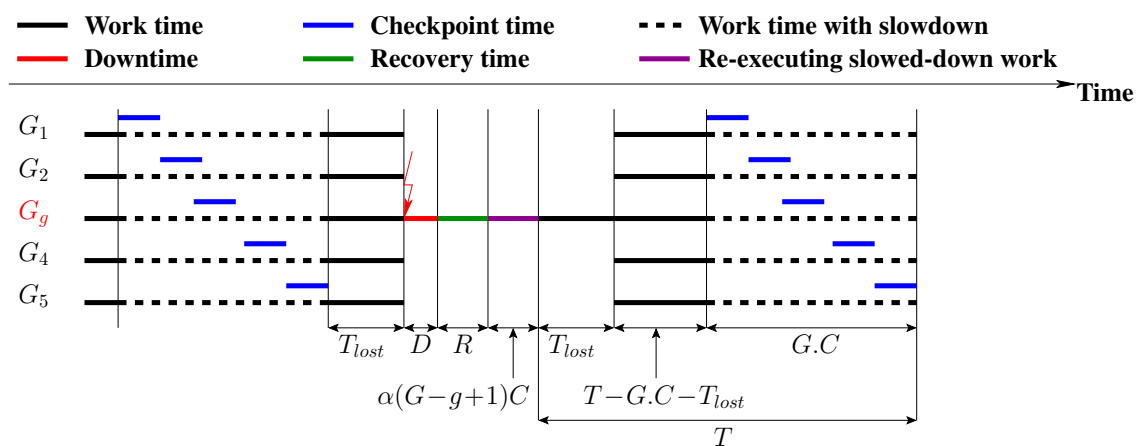

(b)
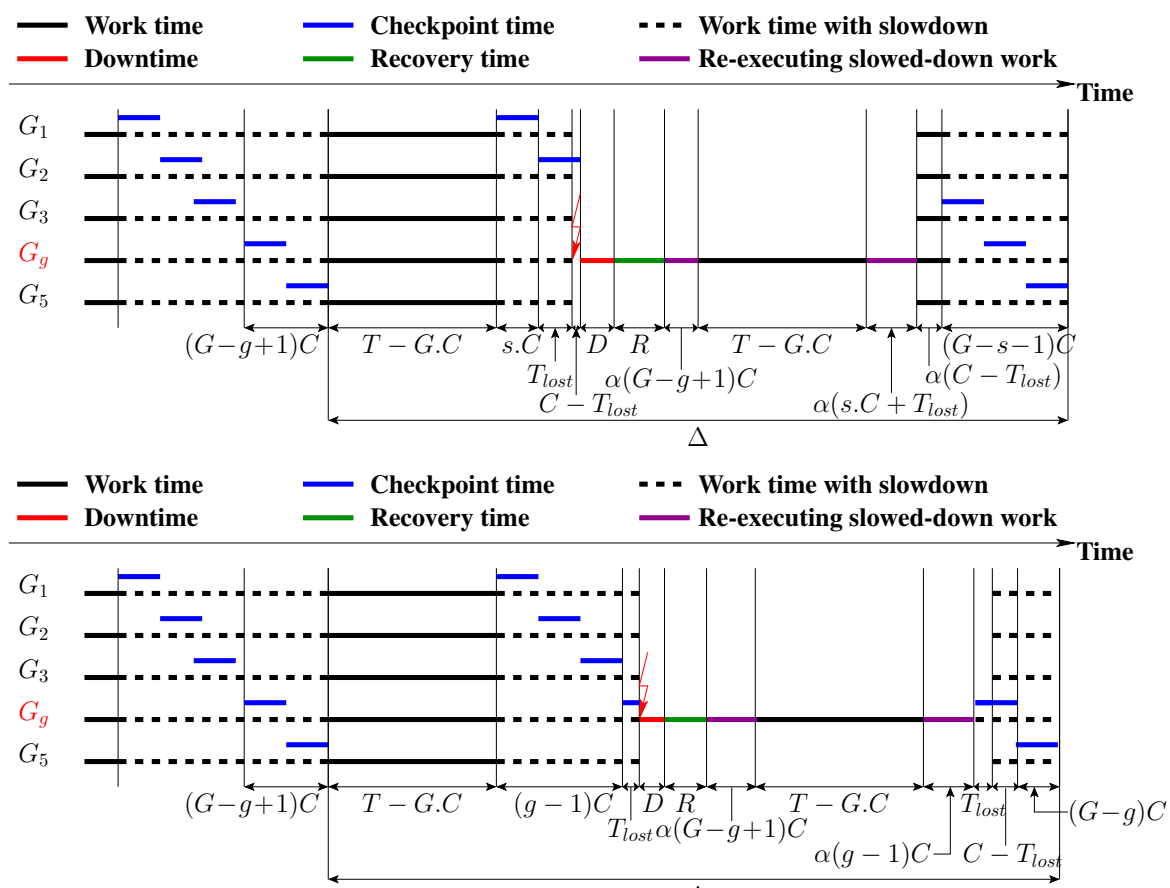

(c)

(d)

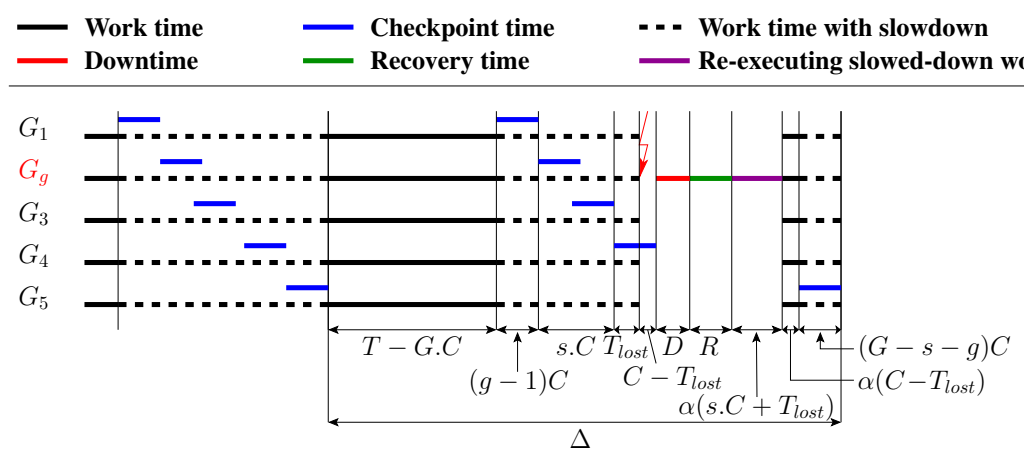

Figure 2. Hierarchical checkpoint: illustrating the waste when a failure occurs (a) during the work phase (Equation (15)); and during the checkpoint phase (Equations (16)-(19)), with three sub-cases: (b) before the checkpoint of the failing group (Equation (17)), (c) during the checkpoint of the failing group

(Equation (18)), or (d) after the checkpoint of the failing group (Equation (19)).

obtain

$$
\text { WASTE }_{\text {hier }}=1-\left(1-\frac{T-\lambda \text { WORK }}{T}\right)\left(1-\frac{1}{\mu_{p}}\left(D(q)+R(q)+\frac{\text { RE-EXEC }}{\rho}\right)\right)
$$


where the values of WORK and RE-EXEC are unchanged, and given by Equations (14) and (15 - 19) respectively.

Impact of message logging on checkpoint size- Message logging has an impact on the execution and re-execution rates, but also on the size of the checkpoint. Because inter-group messages are logged, the size of the checkpoint increases with the amount of work per unit. Consider the hierarchical scenario with $G$ groups of $q$ processors. Without message logging, the checkpoint time of each group is $C_{0}(q)$, and to account for the increase in checkpoint size due to message logging, we write the equation

$$
C(q)=C_{0}(q)(1+\beta \lambda \text { WORK }) \Leftrightarrow \beta=\frac{C(q)-C_{0}(q)}{C_{0}(q) \lambda \text { WORK }}
$$

As before, $\lambda$ WORK $=\lambda(T-(1-\alpha) G C(q))$ (see Equation (14)) is the number of work units, or application iterations, completed during the period of duration $T$, and the parameter $\beta$ quantifies the increase in the checkpoint image size per work unit, as a proportion of the application footprint. Typical values of $\beta$ are given in the examples of Section 4. Combining with Equation (22), we derive the value of $C(q)$ as

$$
C(q)=\frac{C_{0}(q)(1+\beta \lambda T)}{1+G C_{0}(q) \beta \lambda(1-\alpha)}
$$

The first constraint in Equation (20), namely $G C(q) \leq T$, now translates into $\frac{G C_{0}(q)(1+\beta \lambda T)}{1+G C_{0}(q) \beta \lambda(1-\alpha)} \leq$ $T$, hence

$$
G C_{0}(q) \beta \lambda \alpha \leq 1 \text { and } T \geq \frac{G C_{0}(q)}{1-G C_{0}(q) \beta \lambda \alpha}
$$

\section{CASE STUDIES}

In this section, we use the previous model to evaluate different case studies. We propose three generic scenarios for the checkpoint protocols, three application examples with different values for the parameter $\beta$, and four platform instances.

\subsection{Checkpointing algorithm scenarios}

COORD-IO- The first scenario considers a coordinated approach, where the duration of a checkpoint is the time needed for the $p_{\text {total }}$ processors to write the memory footprint of the application onto stable storage. Let Mem denote this memory, and $\mathrm{b}_{i o}$ represents the available I/O bandwidth. Then

$$
C=C_{\mathrm{Mem}}=\frac{\mathrm{Mem}}{\mathrm{b}_{i o}}
$$

In most cases we have equal write and read speed access to stable storage, and we let $R=C=$ $C_{\mathrm{Mem}}$, but in some cases we have different values, for example with the K-Computer (see Table I). As for the downtime, the value $D$ is the expectation of the duration of the downtime. With a single processor, the downtime has a constant value, but with several processors, the duration of the downtime is difficult to compute: a processor can fail while another one is down, thereby leading to cascading downtimes. The exact value of the downtime with several processors is unknown, even for failures distributed according to an exponential law; but in most practical cases, the lower bound given by the downtime of a single processor is expected to be very accurate, and we use a constant value for $D$ in our case studies.

HIERARCH-IO- The second scenario uses a number of relatively large groups. Typically, these groups are composed to take advantage of the application communication pattern [10, 11]. For instance, if the application executes on a 2D-grid of processors, a natural way to create processor groups is to have one group per row (or column) of the grid. If all processors of a given row belong to the same group, horizontal communications are intra-group communications and need not to be logged. Only vertical communications are inter-group communications and need to be logged. 
With large groups, there are enough processors within each group to saturate the available I/O bandwidth, and the $G$ groups checkpoint sequentially. Hence the total checkpoint time without message logging, namely $G C_{0}(q)$, is equal to that of the coordinated approach. This leads to the simple equation

$$
C_{0}(q)=\frac{C_{\mathrm{Mem}}}{G}=\frac{\mathrm{Mem}}{G \mathbf{b}_{i o}}
$$

where Mem denotes the memory footprint of the application, and $\mathrm{b}_{i o}$ the available I/O bandwidth. Similarly as before, we use $R(q)$ for the recovery (either equal to $C(q)$ or not), and a constant value $D(q)=D$ for the downtime.

HIERARCH-PORT- The third scenario investigates the possibility of having a large number of very small groups, a strategy proposed to take advantage of hardware proximity and failure probability correlations [9]. However, if groups are reduced to a single processor, a single checkpointing group is not sufficient to saturate the available I/O bandwidth. In this strategy, multiple groups of $q$ processors are allowed to checkpoint simultaneously in order to saturate the I/O bandwidth. We define $\mathrm{q}_{\min }$ as the smallest value such that $\mathrm{q}_{\min } \mathrm{b}_{\text {port }} \geq \mathrm{b}_{i o}$, where $\mathrm{b}_{\text {port }}$ is the network bandwidth of a single processor. In other words, $\mathrm{q}_{\min }$ is the minimal size of groups so that Equation (26) holds.

Small groups typically imply logging more messages (hence a larger growth factor of the checkpoint per work unit $\beta$, and possibly a larger impact on computation slowdown $\lambda$ ). For an application executing on a 2D-grid of processors, twice as many communications will be logged (assuming a symmetrical communication pattern along each grid direction). However, let us compare recovery times in the HIERARCH-PORT and HIERARCH-IO strategies; assume that $R_{0}(q)=C_{0}(q)$ for simplicity. In both cases Equation (26) holds, but the number of groups is significantly larger for HIERARCH-PORT, thereby ensuring a much shorter recovery time.

\subsection{Application examples}

We study the increase in checkpoint size due to message logging by detailing three application examples that are typical scientific applications executing on 2D-or 3D-processor grids, but that exhibit a different checkpoint increase rate parameter $\beta$.

2D-STENCIL- We first consider a 2D-stencil computation: a real matrix of size $n \times n$ is partitioned across a $p \times p$ processor grid, where $p^{2}=p_{\text {total }}$. At each iteration, each element is averaged with its 8 closest neighbors, requiring rows and columns that lie at the boundary of the partition to be exchanged (it is easy to generalize to larger update masks). Each processor holds a matrix block of size $b=n / p$, and sends four messages of size $b$ (one in each grid direction). Then each element is updated, at the cost of 9 double floating-point operations. The (parallel) work for one iteration is thus WORK $=\frac{9 b^{2}}{\mathrm{~s}_{p}}$, where $\mathrm{s}_{p}$ is the speed of one processor.

Here Mem $=8 n^{2}$ (in bytes), since there is a single (double real) matrix to store. As already mentioned, a natural (application-aware) group partition is with one group per row (or column) of the grid, which leads to $G=q=p$. Such large groups correspond to the HIERARCH-IO scenario, with $C_{0}(q)=\frac{C_{\text {Mem }}}{G}$. At each iteration, vertical (inter-group) communications are logged, but horizontal (intra-group) communications are not logged. The size of logged messages is thus $2 p b=2 n$ for each group. If we checkpoint after each iteration, $C(q)-C_{0}(q)=\frac{2 n}{\mathrm{~b}_{i o}}$, and we derive from Equation (22) that $\beta=\frac{2 n p s_{p}}{n^{2} 9 b^{2}}=\frac{2 s_{p}}{9 b^{3}}$. We stress that the value of $\beta$ is unchanged if groups checkpoint every $k$ iterations, because both $C(q)-C_{0}(q)$ and WORK are multiplied by a factor $k$. Finally, if we use small groups of size $\mathrm{q}_{\text {min }}$, we have the HIERARCH-PORT scenario. We still have $C_{0}(q)=\frac{C_{\mathrm{Mem}}}{G}$, but now the value of $\beta$ has doubled since we log twice as many communications.

MATRIX-PRODUCT-Consider now a typical linear-algebra kernel involving matrix products. For each matrix-product, there are three matrices involved, so Mem $=24 n^{2}$ (in bytes). The matrix partition is similar to previous scenario, but now each processor holds three matrix blocks of size $b=n / p$. Consider Cannon's algorithm [29] which has $p$ steps to compute a product. At each step, each processor shifts one block vertically and one block horizontally, and WORK $=\frac{2 b^{3}}{\mathrm{~s}_{p}}$. In the HIERARCH-IO scenario with one group per grid row, only vertical messages are logged: $\beta=\frac{s_{p}}{6 b^{3}}$. 
Again, $\beta$ is unchanged if groups checkpoint every $k$ steps, or every matrix product $(k=p)$. In the COORD-PORT scenario with groups of size $\mathrm{q}_{\min }$, the value of $\beta$ is doubled.

3D-STENCIL- This application is similar to 2D-STENCIL, but with a 3D matrix of size $n$ partitioned across a 3D-grid of size $p$, where $8 n^{3}=$ Mem and $p^{3}=p_{\text {total }}$. Each processor holds a cube of size $b=n / p$. At each iteration, each pixel is averaged with its 26 closest neighbors, and WORK $=\frac{27 b^{3}}{\mathrm{~s}_{p}}$. Each processor sends the six faces of its cube, one in each direction. In addition to COORD-IO, there are now three hierarchical scenarios: A) HIERARCH-IO-PLANE where groups are horizontal planes, of size $p^{2}$. Only vertical communications are logged, which represents two faces per processor: $\beta=$ $\frac{2 s_{p}}{27 b^{3}}$; B) HIERARCH-IO-LINE where groups are lines, of size $p$. Twice as many communications are logged, which represents four faces per processor: $\beta=\frac{4 \mathrm{~s}_{p}}{27 b^{3}}$; C) HIERARCH-PORT (groups of size $\mathrm{q}_{\text {min }}$ ). All communications are logged, which represents six faces per processor: $\beta=\frac{6 \mathrm{~s}_{p}}{27 b^{3}}$. The order of magnitude of $b$ is the cubic root of the memory per processor for 3D-STENCIL, while it was its square root for 2D-STENCIL and MATRIX-PRODUCT, so $\beta$ will be larger for 3D-STENCIL.

\subsection{Platforms and parameters}

We consider multiple platforms, existing or envisioned, that represent state-of-the-art targets for HPC applications. Table I presents the basic characteristics of the platforms we consider. The machine named Titan represents the fifth phase of the Jaguar supercomputer, as presented by the Oak Ridge Leadership Computing Facility (http://www.olcf.ornl.gov/ computing-resources/titan/). The cumulated bandwidth of the I/O network is targeted to top out at $1 \mathrm{MB} / \mathrm{s} /$ core, resulting in $300 \mathrm{~GB} / \mathrm{s}$ for the whole system. We consider that all existing machines are limited for a single node output by the bus capacity, at approximately $20 \mathrm{~GB} / \mathrm{s}$. The $\mathrm{K}$-Computer machine, hosted by Riken in Japan, is the second fastest supercomputer of the Top 500 list at the time of writing. Its I/O characteristics are those presented during the Lustre File System User's Group meeting, in April, 2011 [30], with the same bus limitation for a single node maximal bandwidth. The two Exascale machines represent the two most likely scenarios envisioned by the International Exascale Software Project community [1], the largest variation being on the number of cores a single node should host. For all platforms, we let the speed of one core be 1 Gigaflops, and we derive the speed of one processor $\mathrm{s}_{p}$ by multiplying by the number of cores.

Table I also presents key parameters for all platform/scenario combinations. In all instances, we use the default values: $\rho=1.5, \lambda=0.98$ and $\alpha=0.3$. These values lead to results representative of the trends observed throughout the set of tested values.

\subsection{Checkpoint duration}

The last parameter that we consider is the duration of the checkpoint. Equation (25) states that $C=C_{\mathrm{Mem}}=\frac{\mathrm{Mem}}{\mathrm{b}_{i o}}$, hence the duration of the checkpoint is proportional to the volume of data written to stable storage, and inversely proportional to the cumulated I/O bandwidth that is available on the platform. As for the volume of data written, it can range from the entire memory available on the platform (for those applications whose footprint is maximal), down to a small percentage of this value. As for the cumulated I/O bandwidth, it can range from the values given in Table I down to a small fraction of these values, if one can use advanced checkpointing techniques, like incremental checkpointing [31,32] to reduce the checkpoint size, or multi-level checkpointing [33], diskless checkpointing [34,35], or new generation hardware, providing local remanent memory [36] to increase the I/O bandwidth. To account for the wide range of both parameters (volume and bandwidth), we propose several scenarios:

$C_{\max }$ In this scenario, which represents the worst case, the duration of a checkpoint is $C_{\max }=C=$ $C_{\mathrm{Mem}}$ as in Equation (25): here we use the values of Table I, both for Mem (the application uses the entire platform memory, and no technique such as incremental checkpointing, or compressive checkpointing, can be used to reduce the amount of memory that needs to be saved), and for $b_{i o}$ (the checkpoint is stored in the remote reliable file storage system, and its transfer speed is limited by the I/O bandwidth of the platform); 
$\frac{C_{\max }}{X}$ In these scenarios, the duration of a checkpoint is $\frac{C_{\max }}{X}$, where $X \in\{10,100,1000\}$. Note that this does not mean that a single technique allows to reduce the data volume by a factor $X$; instead, the ratio $\frac{\mathrm{Mem}}{\mathrm{b}_{i o}}$ is divided by $X$, by combining all available techniques and hardware, and both the numerator (smaller volume) and the denominator (faster transfer) can contribute to the reduction of checkpoint duration. The objective is to investigate whether, and to what extent, faster checkpointing can prove useful, or necessary, at very large scale.

\section{RESULTS FROM THE MODEL}

This section covers the results of our unified model on the previously described scenarios. In order to grant fellow researchers access to the model, results and scenarios proposed in this paper, we made our computation spreadsheet available at http://icl.cs.utk.edu/ herault/ UnifiedModel/.

We start with some words of caution. First, the applications used for this evaluation exhibit properties that makes them a difficult case for hierarchical checkpoint/restart techniques. These applications are communication intensive, which leads to a noticeable impact on performance (due to message logging). In addition, their communication patterns create logical barriers that make them tightly-coupled, giving a relative advantage to all coordinated checkpointing methods (due to the lack of independent progress). However, these applications are more representative of typical HPC applications than loosely-coupled (or even independent) jobs, and their communication-tocomputation ratio tends to zero with the problem size (full weak scalability). Next, we point out that the theoretical values used in the model instances, and summarized in Table I, are overly optimistic, based on the values released by the constructors and not on measured values. Finally, we stress that the horizontal axis of all figures is the processor MTBF $\mu$, which ranges from 1 year to 100 years, a choice consistent with the usual representation in the community.

Section 4 above presented in detail how the values of Table I were obtained. We started with the basic numbers of the different platforms (number of cores, processors, amount of memory and I/O capacity), and derived for each platform and Scenario the corresponding number of groups (for hierarchical protcols), their size, and from this the costs of taking a group checkpoint. We then derived, for each target application, the value of $\beta$, which depends on the group size.

The first observation is that when $C=C_{\max }$, only Titan is a useful platform! Indeed, we obtain a waste equal to 1 for all scenario/application combinations, throughout the whole range of the $\operatorname{MTBF} \mu$, for both the K-Computer or Exascale platforms. This was expected and simply shows that for such large platforms, the checkpoint time must be significantly smaller than $C_{\max }$, the time needed to write the entire platform memory onto stable storage.

Along the same line, we only report values for $C=C_{\max }$ on Titan, for $C=\frac{C_{\max }}{10}$ on Titan and the K-computer, for $C=\frac{C_{\max }}{100}$ on Exascale-Fat, and for $C=\frac{C_{\max }}{1,000}$ on Exascale-Fat and ExascaleSlim. Unreported values correspond to situations where the checkpoint duration is too large for the platform to be useful. A few comments apply to all platforms:

- Hierarchical protocols are very sensitive to message logging: a direct relationship between $\beta$, and the observed waste can be seen when moving from one application to another, and even for different protocols within the same application.

- Hierarchical protocols tend to provide better results for small MTBFs. Thus, they seem more suitable for failure-prone platforms. While they struggle when the communication intensity increases (the case of the 3D-Stencil), they provide limited waste for all the other cases.

- The faster the checkpointing time, the smaller the waste. This conclusion is quite expected, but our results allow quantifying the gain.

On Titan, when using $C_{\max }$, the key factors impacting the balance between coordinated and hierarchical protocols are the communication intensity of the applications (2D-STENCIL, MATRIXPRODUCT, and 3D-STENCIL), and the I/O capabilities of the system. The coordinated protocol has a slow startup, preventing the application from progressing when the platform MTBF $\mu_{p}$ is under a system limit directly proportional to the time required to save the coordinated checkpoint. 
Hierarchical protocols have a faster startup. However, as the MTBF increases, the optimal interval between checkpoints increase, and the cost of logging the messages (and the increase in checkpoint size it implies) becomes detrimental to the hierarchical protocols (even considering the most promising approaches). The vertical segments on the graphs correspond to cut-off values where we enforce the condition $T \leq 0.1 \mu_{p}$ (see Equation (20)). Values of $\mu$ for which no waste is reported correspond to configurations for which no period can satisfy Equation (20).

Moving to $\frac{C_{\max }}{10}$, the same remarks can be made about the shape of the figures. Compared to a checkpointing time of $C_{\max }$, the waste is significantly smaller, leading to a very good yield of the platform as soon as the MTBF $\mu$ exceeds 10 years. The K-Computer shows similar behavior. However, the waste is still important even for large MTBF values for all application scenarios. This can be attributed to the low I/O bandwidth and high amount of total memory of the parameters used for the K-computer, when compared to the parameters considered for the Titan setup.

Moving to Exascale platforms, the Exascale-Fat platform starts to show application progress when $\frac{C_{\max }}{100}$ is used. However, just like the K-Computer, the waste is still important even for large MTBF. When checkpointing becomes ten times faster, the results are more promising. The Exascale-Slim platform starts to be useful when using $\frac{C_{\max }}{1,000}$, which corresponds to checkpointing the application within a few seconds. Overall, Exascale-Fat leads to a smaller waste (or better resource usage) than Exascale-Slim; the main reason is that the Fat version has fewer processors, hence a larger platform MTBF. Indeed, the individual processor MTBF is assumed to be the same for both Exascale-Fat and Exascale-Slim, which may be unfair since there are 10 times more cores per node in the Fat version.

Setting aside the expected conclusion that an efficient process checkpointing strategy will be critical to enable rollback/recovery at exascale, the model leads to an important prediction for Exascale machines (Fat or Slim scenarios): unless extremely high reliability of the components can be guaranteed (MTBF per component of 30 to 50 years for the Stencil applications), hierarchical checkpointing approaches will (i) exhibit a lower waste than coordinated checkpointing, and (ii) allow for an efficiency two to four times higher than replication. This conclusion holds even for applications as tightly-coupled and as communication-intensive as the ones evaluated in this study.

\section{VALIDATION OF THE MODEL}

To validate the mathematical model, we wrote a simulator that generates a random trace of errors (parameterized by an Exponential failure distribution or a Weibull one with a shape parameter of 0.7 or 0.5 ). On this error trace, we simulate the behavior of the various fault tolerance protocols. In the simulator, there is no assumption on when errors can happen: an error can strike a processor while another or the same processor is already subject to a failure and during a recovery phase.

We arbitrarily set the failure-free duration of the parallel application execution to 4 days. This guarantees that enough failures happen during each simulation run to evaluate the waste. We measure the simulated execution time of each application on each platform and on each error trace using a time-out of one year: if an execution does not complete before the one year deadline, we consider it never completes and do not report any result for this particular platform/application setting. From the simulated execution times, we compute the average waste.

All protocols use the same parameters in the simulator as the ones fed to the mathematical model: checkpoint durations, overheads of message logging and consequences in the checkpoint size, amount of work that can be done in parallel, are simulated by increasing accordingly the duration of the execution. The checkpoint interval is set in each case to the optimal value, as provided by the mathematical model. In order to evaluate the accuracy of the optimal checkpoint interval forecasted by the model, we also ran a set of experiments that investigate other random checkpoint interval values around the forecasted best value, and keep the best value in the experiments denoted BestPer.

Figure 4 reports the waste for various application/platform scenarios for a Weibull failure distribution with $k=0.7$. Results for an Exponential failure distribution and for a Weibull with $k=0.5$, are provided at http://icl.cs.utk.edu/ herault/UnifiedModel/. Each point on the graphs is an average over 20 randomly generated instances. 
Overall, Figures 3 and 4 present similar trends and conclusions. The main differences are seen for low MTBFs, in the vicinity of cut-offs values for Figure 3. There, either the first-order assumption could no longer be satisfied, or coordinated protocols were assessed not to allow for any application progress. Simulations show that, in these extreme settings, our analytical study was pessimistic: coordinated protocols have indeed very bad performance, but often applications still make progress (albeit at unsatisfactory rate); coordinated protocols have an advantage over hierarchical protocols for slightly lower MTBFs than predicted. However, the simulations validate the relative performance, and the general efficiency, of the different protocols.

For each scenario and each protocol, we plot (in solid line) the average waste for the checkpointing period computed with our model (the one minimizing Equation (21)). In Figure 4 we also plot (in dotted line) the average waste obtained for the best checkpointing period (BestPer), numerically found by generating, and evaluating through simulations, a set of 480 periods representative of a very large neighborhood of the period computed with our model. The very good adequation between solid and dotted lines show that our model enables to compute near-optimal checkpointing periods, even when its underlying assumptions cannot be guaranteed.

\section{CONCLUSION}

Despite the increasing importance of fault tolerance in achieving sustained, predictable performance, the lack of models and predictive tools has restricted the analysis of fault tolerant protocols to experimental comparisons only, which are painfully difficult to realize in a consistent and repeated manner. This paper introduces a comprehensive model of rollback recovery protocols that encompasses a wide range of checkpoint/restart protocols, including coordinated checkpoint and an assortment of uncoordinated checkpoint protocols (based on message logging). This model provides the first tool for a quantitative assessment of all these protocols. Instantiation on future platforms enables the investigation and understanding of the behavior of fault tolerant protocols at scales currently inaccessible. The results presented in Section 5, and corroborated by Section 6, highlight the following tendencies:

- Hardware properties will have tremendous impact on the efficiency of future platforms. Under the early assumptions of the projected Exascale systems, rollback recovery protocols are mostly ineffective. In particular, significant efforts are required in terms of $\mathrm{I} / \mathrm{O}$ bandwidth to enable any type of rollback recovery to be competitive. With the appropriate provision in I/O (or the presence of distributed storage on nodes), rollback recovery can be competitive and significantly outperform full-scale replication [3] (which by definition cannot reach more than $50 \%$ efficiency).

- Under the assumption that I/O network provision is sufficient, the reliability of individual processors has a minor impact on rollback recovery efficiency. This suggests that most research efforts, funding and hardware provisions should be directed to I/O performance rather than improving component reliability in order to increase the scientific throughout of Exascale platforms. - The model outlines some realistic ranges where hierarchical checkpointing outperforms coordinated checkpointing, thanks to its faster recovery from individual failures. This early result had already been outlined experimentally at smaller scales, but it was difficult to project at future scales. Our study provides a theoretical foundation and a quantitative evaluation of the drawbacks of checkpoint/restart protocols at Exascale; it can be used as a first building block to drive the research field forward, and to design platforms with specific resilience requirements.

Throughout the simulations, we have checked (by an extensive brute-force comparison) that our model could predict near-optimal checkpointing periods for the whole range of the protocol/platform/application combinations; this gives us very good confidence that this model will prove reliable and accurate in other frameworks. As we are far from a comprehensive understanding of future Exascale applications and platform characteristics, we hope that the community will be interested in instantiating our publicly available model with different scenarios and case-studies. 


\section{ACKNOWLEDGEMENTS}

This work was partly supported by the French ANR White Project RESCUE, the US National Science Foundation through award \#1063019, and the US Department Of Energy Award \#DEFG02-11ER26059. Yves Robert is with Institut Universitaire de France. We would like to thank the reviewers for their comments and suggestions, which greatly helped improve the final version of the paper. 


\begin{tabular}{|c|c|c|c|c|}
\hline Name & $\begin{array}{c}\text { Number of } \\
\text { cores }\end{array}$ & $\begin{array}{c}\text { Number of } \\
\text { processors } p_{\text {total }}\end{array}$ & $\begin{array}{c}\text { Number of cores } \\
\text { per processor }\end{array}$ & $\begin{array}{c}\text { Memory } \\
\text { per processor }\end{array}$ \\
\hline Titan & 299,008 & 18,688 & 16 & $32 \mathrm{~GB}$ \\
\hline K-Computer & 705,024 & 88,128 & 8 & $16 \mathrm{~GB}$ \\
\hline Exascale-Slim & $1,000,000,000$ & $1,000,000$ & 1,000 & $64 \mathrm{~GB}$ \\
\hline Exascale-Fat & $1,000,000,000$ & 100,000 & 10,000 & $640 \mathrm{~GB}$ \\
\hline
\end{tabular}

\begin{tabular}{|c|c|c|c|}
\hline \multirow[t]{2}{*}{ Name } & \multicolumn{2}{|c|}{ I/O Network Bandwidth $\left(\mathrm{b}_{i o}\right)$} & I/O Bandwidth ( $\mathrm{b}_{\text {port }}$ ) \\
\hline & Read & Write & Read/Write per processor \\
\hline Titan & $300 \mathrm{~GB} / \mathrm{s}$ & $300 \mathrm{~GB} / \mathrm{s}$ & $20 \mathrm{~GB} / \mathrm{s}$ \\
\hline K-Computer & $150 \mathrm{~GB} / \mathrm{s}$ & $96 \mathrm{~GB} / \mathrm{s}$ & $20 \mathrm{~GB} / \mathrm{s}$ \\
\hline Exascale-Slim & $1 \mathrm{~TB} / \mathrm{s}$ & $1 \mathrm{~TB} / \mathrm{s}$ & $200 \mathrm{~GB} / \mathrm{s}$ \\
\hline Exascale-Fat & $1 \mathrm{~TB} / \mathrm{s}$ & $1 \mathrm{~TB} / \mathrm{s}$ & $400 \mathrm{~GB} / \mathrm{s}$ \\
\hline
\end{tabular}

\begin{tabular}{|c|c|c|c|c|}
\hline Name & Scenario & $G(C(q))$ & $\begin{array}{c}\beta \text { for } \\
\text { 2D-STENCIL }\end{array}$ & $\begin{array}{c}\beta \text { for } \\
\text { MATRIX-PRODUCT }\end{array}$ \\
\hline \multirow{3}{*}{ Titan } & COORD-IO & $1(2,048 \mathrm{~s})$ & $/$ & $/$ \\
& HIERARCH-IO & $136(15 \mathrm{~s})$ & 0.0001098 & 0.0004280 \\
& HIERARCH-PORT & $1,246(1.6 \mathrm{~s})$ & 0.0002196 & 0.0008561 \\
\hline \multirow{3}{*}{ K-Computer } & COORD-IO & $1(14,688 \mathrm{~s})$ & $/$ & $/$ \\
& HIERARCH-IO & $296(50 \mathrm{~s})$ & 0.0002858 & 0.001113 \\
& HIERARCH-PORT & $17,626(0.83 \mathrm{~s})$ & 0.0005716 & 0.002227 \\
\hline \multirow{3}{*}{ Exascale-Slim } & COORD-IO & $1(68,719 \mathrm{~s})$ & $/$ & $/$ \\
& HIERARCH-IO & $1,000(68.7 \mathrm{~s})$ & 0.0002599 & 0.001013 \\
& HIERARCH-PORT & $200,000(0.32 \mathrm{~s})$ & 0.0005199 & 0.002026 \\
\hline \multirow{3}{*}{ Exascale-Fat } & COORD-IO & $1(68,719 \mathrm{~s})$ & $/$ & $/$ \\
& HIERARCH-IO & $316(217 \mathrm{~s})$ & 0.00008220 & 0.0003203 \\
& HIERARCH-PORT & $33,333(1.92 \mathrm{~s})$ & 0.00016440 & 0.0006407 \\
\hline
\end{tabular}

\begin{tabular}{|c|c|c|c|}
\hline Name & ScenariO & $G$ & $\beta$ for 3D-STENCIL \\
\hline \multirow{5}{*}{ Titan } & COORD-IO & 1 & $/$ \\
& HIERARCH-IO-PLANE & 26 & 0.001476 \\
& HIERARCH-IO-LINE & 676 & 0.002952 \\
& HIERARCH-PORT & 1,246 & 0.004428 \\
\hline \multirow{3}{*}{ K-Computer } & COORD-IO & 1 & $/$ \\
& HIERARCH-IO-PLANE & 44 & 0.003422 \\
& HIERARCH-IO-LINE & 1,936 & 0.006844 \\
& HIERARCH-PORT & 17,626 & 0.010266 \\
\hline \multirow{3}{*}{ Exascale-Slim } & COORD-IO & 1 & $/$ \\
& HIERARCH-IO-PLANE & 100 & 0.003952 \\
& HIERARCH-IO-LINE & 10,000 & 0.007904 \\
& HIERARCH-PORT & 200,000 & 0.011856 \\
\hline \multirow{3}{*}{ Exascale-Fat } & COORD-IO & 1 & \\
& HIERARCH-IO-PLANE & 46 & 0.001834 \\
& HIERARCH-IO-LINE & 2,116 & 0.003668 \\
& HIERARCH-PORT & 33,333 & 0.005502 \\
\hline
\end{tabular}

Table I. Basic characteristics of platforms used to instantiate the model, and all parameters for each platform/scenario combination. The equations $C_{0}(q)=C / G$ and $R_{0}(q)=R / G$ always hold. 

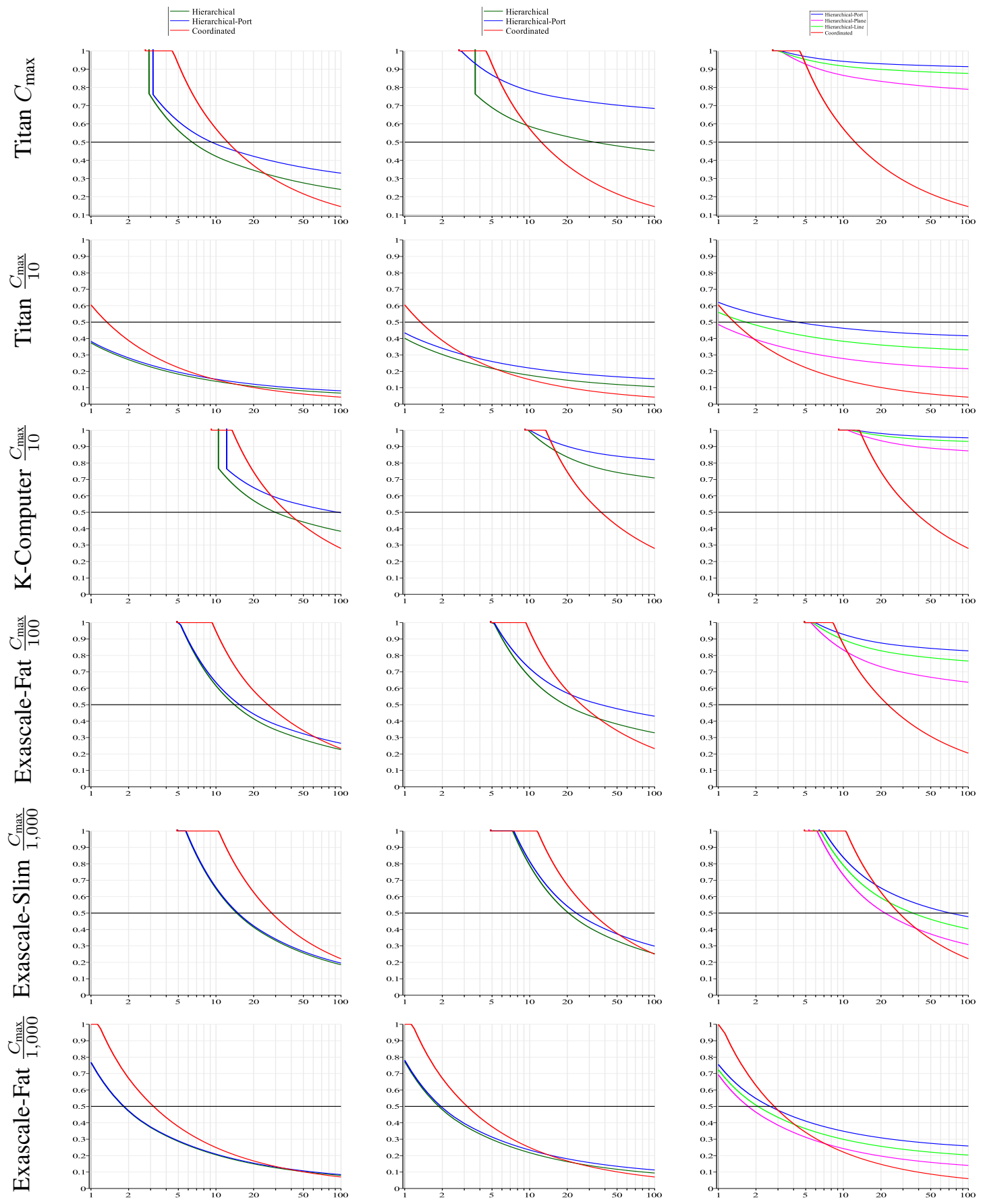

2D-STENCIL

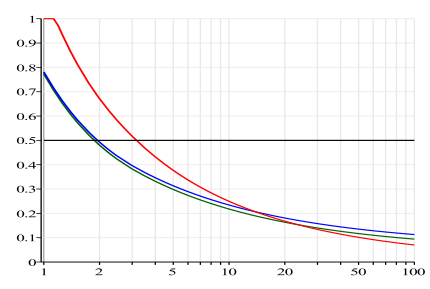

MATRIX-PRODUCT

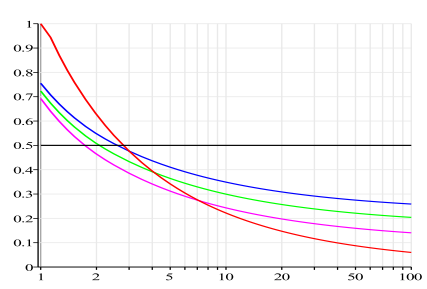

3D-STENCIL

Figure 3. Model: waste as a function MTBF $\mu$ (years per processor). 

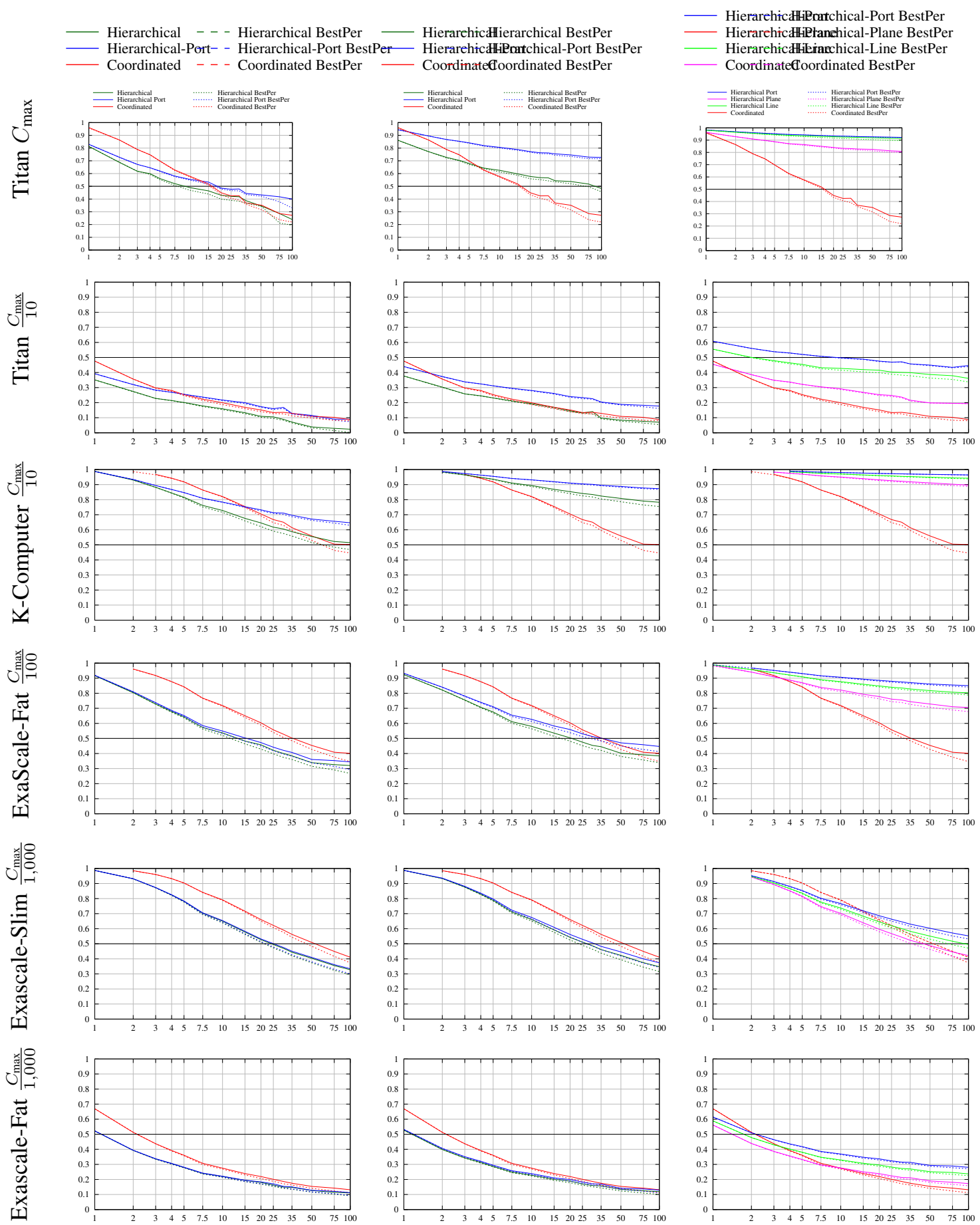

2D-STENCIL
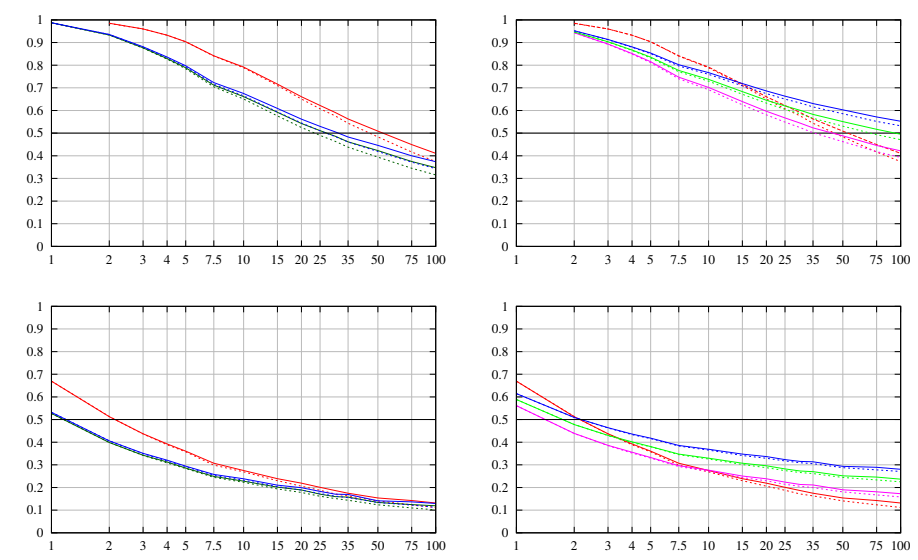

MATRIX-PRODUCT

3D-STENCIL

Figure 4. Waste as a function of processor $\operatorname{MTBF} \mu$, for a Weibull distribution with $\mathrm{k}=0.7$ 
References

1. Dongarra J, Beckman P, Aerts P, Cappello F, Lippert T, Matsuoka S, Messina P, Moore T, Stevens R, Trefethen A, et al.. The international exascale software project: a call to cooperative action by the global highperformance community. Int. J. High Perform. Comput. Appl. 2009; 23(4):309-322, doi:http://dx.doi.org/10.1177/ 1094342009347714.

2. Sarkar V, et al. Exascale software study: Software challenges in extreme scale systems 2009. White paper available at: http://users.ece.gatech.edu/mrichard/ExascaleComputingstudyReports/ ECSS20report:20101909.pdf.

3. Ferreira K, Stearley J, Laros JHI, Oldfield R, Pedretti K, Brightwell R, Riesen R, Bridges PG, Arnold D. Evaluating the Viability of Process Replication Reliability for Exascale Systems. Proc. ACM/IEEE Conf. on Supercomputing, 2011.

4. Bouteiller A, Herault T, Krawezik G, Lemarinier P, Cappello F. MPICH-V: a multiprotocol fault tolerant MPI. IJHPCA 2006; 20(3):319-333, doi:10.1177/1094342006067469.

5. Rao S, Alvisi L, Viny HM, Sciences DC. Egida: An extensible toolkit for low-overhead fault-tolerance. In Symposium on Fault-Tolerant Computing, Press, 1999; 48-55.

6. Elnozahy ENM, Alvisi L, Wang YM, Johnson DB. A survey of rollback-recovery protocols in message-passing systems. ACM Computing Survey 2002; 34:375-408.

7. Chandy KM, Lamport L. Distributed snapshots : Determining global states of distributed systems. Transactions on Computer Systems, vol. 3(1), ACM, 1985; 63-75.

8. Rao S, Alvisi L, Vin HM. The cost of recovery in message logging protocols. 17th Symposium on Reliable Distributed Systems (SRDS), IEEE CS Press, 1998; 10-18.

9. Bouteiller A, Herault T, Bosilca G, Dongarra JJ. Correlated set coordination in fault tolerant message logging protocols. Proc. of Euro-Par'11 (II), LNCS, vol. 6853, Springer, 2011; 51-64, doi:http://dx.doi.org/10.1007/ 978-3-642-23397-5_6.

10. Guermouche A, Ropars T, Snir M, Cappello F. HydEE: Failure Containment without Event Logging for Large Scale Send-Deterministic MPI Applications. IPDPS'12, IEEE, 2012.

11. Esteban Meneses CLM, Kalé LV. Team-based message logging: Preliminary results. Workshop Resilience in Clusters, Clouds, and Grids (CCGRID 2010)., 2010.

12. Young JW. A first order approximation to the optimum checkpoint interval. Comm. of the ACM 1974; 17(9):530 531.

13. Daly JT. A higher order estimate of the optimum checkpoint interval for restart dumps. FGCS 2004; 22(3):303-312.

14. Ling Y, Mi J, Lin X. A variational calculus approach to optimal checkpoint placement. IEEE Trans. Computers 2001; :699-708

15. Ozaki T, Dohi T, Okamura H, Kaio N. Distribution-free checkpoint placement algorithms based on min-max principle. IEEE TDSC 2006; :130-140.

16. Bougeret M, Casanova H, Rabie M, Robert Y, Vivien F. Checkpointing strategies for parallel jobs. Proc. ACM/IEEE Conf. on Supercomputing, 2011.

17. Plank JS, Thomason MG. Processor allocation and checkpoint interval selection in cluster computing systems. Journal of Parallel and Distributed Computing 2001; 61:1590.

18. Jin H, Chen Y, Zhu H, Sun XH. Optimizing HPC Fault-Tolerant Environment: An Analytical Approach. 39th Int. Conf. Parallel Processing (ICPP), 2010; 525 -534, doi:10.1109/ICPP.2010.80.

19. Wang L, Karthik P, Kalbarczyk Z, Iyer R, Votta L, Vick C, Wood A. Modeling Coordinated Checkpointing for Large-Scale Supercomputers. Proceedings of ICDSN'05, 2005; 812-821.

20. Oldfield R, Arunagiri S, Teller P, Seelam S, Varela M, Riesen R, Roth P. Modeling the impact of checkpoints on next-generation systems. Proceedings of IEEE MSST'07, 2007; 30 -46, doi:10.1109/MSST.2007.4367962.

21. Zheng Z, Lan Z. Reliability-aware scalability models for high performance computing. Proc. of IEEE Cluster'09, 2009; 1 -9, doi:10.1109/CLUSTR.2009.5289177.

22. Bouguerra MS, Trystram D, Wagner F. Complexity Analysis of Checkpoint Scheduling with Variable Costs. IEEE Transactions on Computers 2012; 99(PrePrints), doi:http://doi.ieecomputersociety.org/10.1109/TC.2012.57.

23. Wu M, Sun XH, Jin H. Performance under failures of high-end computing. Proc. ACM/IEEE Conf. on Supercomputing, 2007.

24. Heath T, Martin RP, Nguyen TD. Improving cluster availability using workstation validation. SIGMETRICS Perf. Eval. Rev. 2002; 30(1):217-227.

25. Schroeder B, Gibson GA. A large-scale study of failures in high-performance computing systems. Proc. of DSN, 2006.

26. Liu Y, Nassar R, Leangsuksun C, Naksinehaboon N, Paun M, Scott S. An optimal checkpoint/restart model for a large scale high performance computing system. IPDPS'08, IEEE, 2008; 1-9.

27. Heien E, Kondo D, Gainaru A, LaPine D, Kramer B, Cappello F. Modeling and tolerating heterogeneous failures in large parallel systems. Proc. ACM/IEEE Conf. on Supercomputing, 2011.

28. Bouteiller A, Bosilca G, Dongarra J. Redesigning the message logging model for high performance. Concurrency and Computation: Practice and Experience 2010; 22(16):2196-2211, doi:10.1002/cpe.1589.

29. Cannon LE. A cellular computer to implement the Kalman filter algorithm. PhD Thesis, Montana State University 1969.

30. Sumimoto S. An Overview of Fujitsu's Lustre Based File System. Lustre Filesystem Users' Group Meeting, Orlando, USA. April 2011

31. Agarwal S, Garg R, Gupta MS, Moreira JE. Adaptive incremental checkpointing for massively parallel systems. Proceedings of the 18th annual international conference on Supercomputing, ICS '04, ACM: New York, NY, USA, 2004; 277-286, doi:10.1145/1006209.1006248.

32. Gioiosa R, Sancho JC, Jiang S, Petrini F, Davis K. Transparent, incremental checkpointing at kernel level: a foundation for fault tolerance for parallel computers. Proceedings of the 2005 ACM/IEEE conference on 
Supercomputing, SC '05, IEEE Computer Society: Washington, DC, USA, 2005; 9-, doi:10.1109/SC.2005.76.

33. Moody A, Bronevetsky G, Mohror K, Supinski BRd. Design, Modeling, and Evaluation of a Scalable Multi-level Checkpointing System. Proceedings of the ACM/IEEE SC Conference, 2010; 1-11.

34. Plank JS, Li K, Puening MA. Diskless checkpointing. IEEE Transactions on Parallel and Distributed Systems 1998; 9:972-986, doi:http://doi.ieeecomputersociety.org/10.1109/71.730527.

35. Gomez LAB, Maruyama N, Cappello F, Matsuoka S. Distributed diskless checkpoint for large scale systems. Proceedings of the 2010 10th IEEE/ACM International Conference on Cluster, Cloud and Grid Computing, CCGRID '10, IEEE Computer Society: Washington, DC, USA, 2010; 63-72, doi:10.1109/CCGRID.2010.40.

36. Ouyang X, Marcarelli S, Panda DK. Enhancing checkpoint performance with staging io and ssd. Proceedings of the 2010 International Workshop on Storage Network Architecture and Parallel I/Os, SNAPI '10, IEEE Computer Society: Washington, DC, USA, 2010; 13-20, doi:10.1109/SNAPI.2010.10. URL http: // dx. doi .org/10. 1109/SNAPI.2010.10. 